\title{
Cannabinoid Modulation of Amygdala Reactivity to Social Signals of Threat in Humans
}

\author{
K. Luan Phan, ${ }^{1}$ Mike Angstadt, ${ }^{1}$ Jamie Golden, ${ }^{2}$ Ikechukwu Onyewuenyi, ${ }^{3}$ Ana Popovska, ${ }^{4}$ and Harriet de Wit ${ }^{2}$ \\ ${ }^{1}$ Department of Psychiatry, University of Michigan and Veterans Affairs Ann Arbor Healthcare System, Ann Arbor, Michigan 48109, ${ }^{2}$ Human Behavioral \\ Pharmacology Laboratory, Department of Psychiatry, University of Chicago, Chicago, Illinois 60637, ${ }^{3}$ Howard University, Washington, DC 20059, and \\ ${ }^{4}$ Amherst College, Amherst, Massachusetts 01002
}

The cannabinoid (CB) system is a key neurochemical mediator of anxiety and fear learning in both animals and humans. The anxiolytic effects of $\Delta^{9}$-tetrahydrocannabinol (THC), the primary psychoactive ingredient in cannabis, are believed to be mediated through direct and selective agonism of $\mathrm{CB}_{1}$ receptors localized within the basolateral amygdala, a critical brain region for threat perception. However, little is known about the effects of THC on amygdala reactivity in humans. We used functional magnetic resonance imaging and a well validated task to probe amygdala responses to threat signals in 16 healthy, recreational cannabis users after a double-blind crossover administration of THC or placebo. We found that THC significantly reduced amygdala reactivity to social signals of threat but did not affect activity in primary visual and motor cortex. The current findings fit well with the notion that THC and other cannabinoids may have an anxiolytic role in central mechanisms of fear behaviors and provide a rationale for exploring novel therapeutic strategies that target the cannabinoid system for disorders of anxiety and social fear.

Key words: THC; cannabinoid; fMRI; amygdala; fear; anxiety

\section{Introduction}

Derivatives of the marijuana plant, Cannabis sativa, have been used medicinally and recreationally for thousands of years. Recently, the identification of endogenous cannabinoids as an important neurotransmitter system with CNS functions has led to rapid advances of knowledge. One function that has been identified for the central cannabinoid system is its role in the regulation of anxiety and fear learning in animals (Haller et al., 2002; Marsicano et al., 2002; Martin et al., 2002; Chhatwal et al., 2005; Viveros et al., 2005). In humans, ingestion of $\Delta^{9}$ tetrahydrocannabinol (THC), the primary psychoactive ingredient in cannabis and a selective cannabinoid receptor $\left(\mathrm{CB}_{1}\right)$ agonist, modulates subjective anxiety (Sethi et al., 1986; Wachtel et al., 2002; D'Souza et al., 2004). $\mathrm{CB}_{1}$ receptors are expressed in high levels in the amygdala, a region strongly linked with fear responses (Katona et al., 2001; Pistis et al., 2004), and thus could account for the striking effects of THC on emotion in both nonhuman models and in humans.

Core emotional processes such as anxiety and fear learning are dependent on the amygdala (LeDoux, 2000; Phelps, 2004). On perception of threat, the lateral nucleus of the amygdala is

\footnotetext{
Received Sept. 25, 2007; revised Jan. 21, 2008; accepted Jan. 21, 2008.

This work was supported in part by the Brain Research Imaging Center at University of Chicago and National Institutes of Health (NIH) Grants MH076198 (K.L.P.), DA024197 (K.L.P), DA002812, and DA009133 (H.d.W.); the Summer Research Opportunities Program-McNair Scholars' Program (I.0.) of University of Chicago; and Undergraduate Training in Computational Neuroscience NIH Grant DA023425 (A.P.). We thank Jolan Terner, Dan Fitzgerald, and Loren Miller for their assistance in stimulus preparation and data collection.

Correspondence should be addressed to Dr. K. Luan Phan, Department of Psychiatry, University of Michigan, Rachel Upjohn Building, 4250 Plymouth Road, Box 5765, Ann Arbor, MI 48109. E-mail: luan@umich.edu. D01:10.1523/JNEUROSCI.5603-07.2008

Copyright $\odot 2008$ Society for Neuroscience $\quad$ 0270-6474/08/282313-07\$15.00/0
}

thought to integrate inputs and excite the central nucleus to evoke fear responses (LeDoux, 2000). In humans, detection of social signals of threat (e.g., fearful/angry faces) enhances amygdala reactivity (Morris et al., 1996; Whalen et al., 1998), and individuals with pathological anxiety exhibit exaggerated amygdala reactivity to threatening faces (Rauch et al., 2000; Phan et al., 2006). In both monkeys and humans, discrete lesions to the amygdala lead to altered fear responses and perception (Kalin et al., 2001; Adolphs et al., 2005).

There is evidence to suggest that cannabinoids act on anxiety responses and fear learning through their effects on the amygdala. Central $\mathrm{CB}_{1}$ receptors are expressed at high levels in the lateral and basal nuclei of the amygdala (Katona et al., 2001), and extinction of aversive memories depends on cannabinoid receptors and signaling within the basolateral amygdala (Marsicano et al., 2002; Kamprath et al., 2006; Laviolette and Grace, 2006). Activation of $\mathrm{CB}_{1}$ receptors attenuates the anxiety responses and amygdala activation to aversive stimuli (Patel et al., 2005), by modulating neuronal firing in the basolateral amygdala (Pistis et al., 2004). Despite these suggestive lines of evidence in nonhumans, however, the neurocircuitry that underlies the effects of cannabinoids on anxiety in humans remains unknown.

To investigate the neural mechanism underlying the anxiolytic responses to cannabinoids in humans, we examined the effects of THC on threat-related amygdala activation. We used functional magnetic resonance imaging (fMRI) to study acute responses to oral THC (7.5 $\mathrm{mg}$ ) or placebo in healthy volunteers $(n=16)$ and examined amygdala reactivity to social signals of threat (fearful and angry faces), in a double-blind, randomized, placebo-controlled design. The fMRI "activation" task was the Emotional Face Processing Task, a well validated probe of amyg- 
dala reactivity to social threat that has proven useful as a bioassay of pharmacologic (Paulus et al., 2005) and genetic (Hariri et al., 2005) modulation of pathological anxiety (Ressler and Mayberg, 2007).

\section{Materials and Methods}

Subjects. Sixteen healthy, right-handed volunteers (eight males; aged $18-28$ years) participated in this study. All participants had some history of marijuana use but no history or signs of neurological, psychiatric (including substance and alcohol abuse/dependence), or medical illness as confirmed by medical examination and structured clinical interview. Subjects were included if they had used marijuana at least 10 times in their lives but were excluded if they were daily marijuana users. None of the subjects had a positive urine toxicology or alcohol breathalyzer screen at the time of scanning. All participants gave written informed consent after explanation of the experimental protocol, as approved by the University of Chicago Institutional Review Board.

Experimental protocol. This study used a two-session, double-blind, placebo-controlled, within-subject design. The two sessions were scheduled at least 1 week apart. Participants were instructed not to eat for $2 \mathrm{~h}$ before coming to the laboratory and to abstain from all drugs for $24 \mathrm{~h}$ before and $12 \mathrm{~h}$ after each session. We designed the timing of fMRI experiment to match the expected time window for peak subjective effects and plasma levels of THC (Wachtel et al., 2002). Approximately 120 min before beginning the fMRI tasks, participants ingested an opaque, colored gelatin capsule (size 00) with dextrose filler that contained either $\Delta^{9}$-THC (Marinol; $7.5 \mathrm{mg}$; Solvay Pharmaceuticals, Marietta, GA) or placebo (PBO) (containing only dextrose) in random order. This moderate $\Delta^{9}$-THC dose has been previously shown to produce behavioral and subjective effects (Kirk and de Wit, 1999; Wachtel et al., 2002). Based on previous work and our main hypothesis, we were primarily interested in the following dependent psychological measures: Drug Effects Questionnaire (DEQ) (Johanson and Uhlenhuth, 1980) and Visual Analog Scales (VAS) (Folstein and Luria, 1973). The DEQ contained four 100 $\mathrm{mm}$ visual analog scales that the subjects used to mark their response the following questions: (1) Do you feel any drug effects (rated from "none at all" to "a lot")? (2) Do you like the effects you are feeling now (rated from "dislike" to "like very much")? (3) Are you high (rated from "not at all" to "very")? (4) Would you like more of what you consumed, right now (rated from "not at all" to "very much")? The VAS consists of five adjectives and visual analog scales: "stimulated," "high (as in drug high)," "anxious," "sedated," and "hungry"; subjects were required to rate on a 10 -point scale (1, not at all; 10 , extremely) the extent to which they felt each adjective. These affect/drug effect questionnaires were collected 30 min before and at 30, 90, 210, and $270 \mathrm{~min}$ (T1-T5) after ingestion of the $\Delta \Delta^{9}$-THC or placebo capsule.

Approximately $120 \mathrm{~min}$ after ingestion of capsule, the experimental runs of fMRI session began (PBO condition, $125.63 \pm 11.49 \mathrm{~min}$; THC condition, $128.75 \pm 11.28 \mathrm{~min}$ ). First, participants performed a variant of the Emotional Face Processing Task (Hariri et al., 2002a). This task has been previously shown to reliably and robustly engage the amygdala and has been used in other pharmacological fMRI studies (Hariri et al., 2002b; Tessitore et al., 2002; Kirsch et al., 2005; Paulus et al., 2005). In brief, in the emotion task, participants viewed a trio of faces and selected one of the two faces (bottom) that expressed the same emotion as the target face (top). The identity of all three faces was always different, and an equal number of male and female faces were presented. The target and congruent probe face displayed one of three expressions (angry, fearful, or happy), and the other (incongruent) probe face always displayed a neutral/nonemotional expression. This design allowed us to isolate amygdala reactivity specifically to social threat (angry and fearful faces) relative to nonthreat (happy faces), which have similar perceptual characteristics except for the threat/nonthreat signal conveyed. The face photographs were selected from the validated stimulus set from Gur et al. (2002). Of note, the angry, fearful, and happy target faces were presented in separate blocks. Three blocks of each target expression were presented, and no target stimuli were repeated within or across blocks. To maintain attention and allow limbic brain responses to return to baseline, the face matching tasks were interspersed with a "baseline" task, in which subjects matched simple geometric shapes (circles, rectangles, or triangles) similar to instructions above. The paradigm consisted of 18 experimental $20 \mathrm{~s}$ blocks: 9 blocks of matching emotional faces, interleaved with 9 blocks of matching shapes, counterbalanced across two runs for total task time of $6 \mathrm{~min}$. Each task block contained four sequential matching trials, $5 \mathrm{~s}$ each. Participants responded to tasks by pressing the left or right response buttons with their dominant hand. These responses also provided a measure of participants' response accuracy and reaction time.

Second, participants performed a simple visual-motor task, separate from the emotion task above. This visual-motor task was used to engage and isolate activation of the primary visual and motor cortex, in order for us to examine the effects of THC on regional activity outside of the amygdala and unrelated to emotional processing. The visual-motor task consisted of alternating on-off $20 \mathrm{~s}$ blocks during which subjects either viewed a flashing checkerboard while pressing their right index finger rhythmically ("on") or viewed a blank gray screen with a fixation crosshair and remained motionless ("off"); this sequence consisted of six on and eight off epochs over two runs for a total task time of $4 \mathrm{~min}$ and $40 \mathrm{~s}$.

Functional imaging: acquisition and analysis. $\mathrm{PMRI}$ was performed on a 3T GE magnetic resonance scanner at University of Chicago Brain Research Imaging Center. Functional images [i.e., blood oxygenated leveldependent (BOLD)] were collected from 30 axial, 5-mm-thick slices using a $\mathrm{T} 2^{*}$-sensitive gradient echo reverse spiral acquisition sequences (repetition time, $2000 \mathrm{~ms}$; echo time, $25 \mathrm{~ms} ; 64 \times 64$ matrix; $24 \mathrm{~cm}$ field of view; flip angle, 77), optimized to minimize susceptibility artifacts in the amygdala (Stenger et al., 2000). This was followed by a highresolution, T1-weighted volumetric anatomical scan (three-dimensional magnetization-prepared rapid gradient echo) for anatomical localization.

Data from all 16 subjects met criteria for high quality and scan stability with minimum motion correction $(<3 \mathrm{~mm}$ displacement in any one direction) and were subsequently included in fMRI analyses. The first four volumes from each run were discarded to allow for T1 equilibration effects. Functional data were analyzed using SPM2 (Welcome Department of Cognitive Neurology, London, UK; http://www.fil.ion. ucl.ac.uk/spm) and described previously (Fitzgerald et al., 2006). In brief, images were spatially realigned to correct for head motion, warped to an EPI template in Montreal Neurologic Institute (MNI) space, resampled to $2 \mathrm{~mm}^{3}$ voxels, and smoothed with an $8 \mathrm{~mm}^{3}$ kernel to minimize noise and residual differences in gyral anatomy. The general linear model was applied to the time series, convolved with the canonical hemodynamic response function (Friston et al., 1995) and with a $128 \mathrm{~s} \mathrm{high-pass} \mathrm{filter.}$ Condition effects were modeled with box-car regressors representing the occurrence of each block type, and effects were estimated at each voxel, and for each subject. Individual contrast maps [SPMs (statistical parametric maps)] were then analyzed at the second level in a random-effects statistical model (Holmes and Friston, 1998). First, using linear contrasts, we compared amygdala response during angry/fearful face processing relative to happy face (threat vs nonthreat) processing in the placebo session to confirm amygdala activation was present and detectable during the nondrug session and to allow for inferences of THC-induced attenuation of BOLD signal increases. Significant emotion-specific activations of the amygdala were then analyzed in a repeated-measures statistical model. A statistical image for the contrast of social threat versus nonthreat was obtained for each stimulus type and analyzed in a second-level random-effects model (ANOVA) for significant differences in amygdala activation between the THC and PBO condition. The threshold for significance was set to $p<0.05$, corrected for multiple voxelwise comparisons across the amygdala region of interest (ROI) (Worsley et al., 1996), as defined anatomically by atlas (Walter et al., 2003). In addition, to obviate bias, we extracted BOLD signal responses (parameter estimates, $\beta$ weights) of threat versus nonthreat activation from an anatomically based ROI in THC and PBO sessions; in other words, the signal was extracted from an anatomical-based amygdala ROI (Walter et al., 2003) based on masks from the atlas of TzourioMazoyer et al. (2002) (see supplemental Fig. 1, available at www. jneurosci.org as supplemental material). The amygdala search volume comprised $2202 \mathrm{~mm}^{3}$ voxels $\left(\sim 1.8 \mathrm{~cm}^{3}\right)$ on each side (left, right). The 

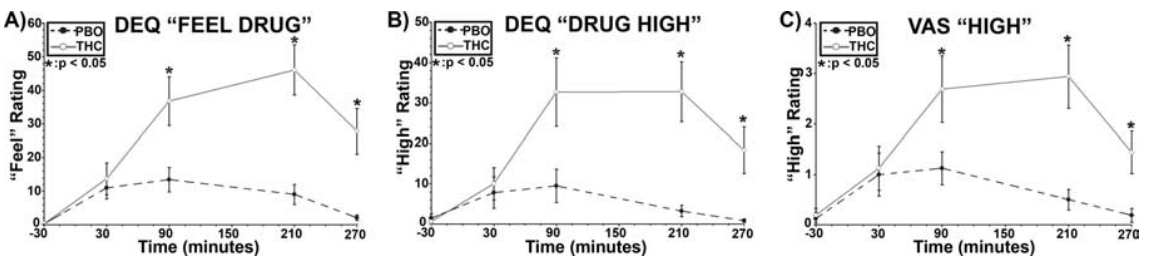

Figure 1. THC effects on self-report measures. Mean "feel drug" (DEQ) (A), "drug high" (DEQ) (B), and "high" (VAS) (C) scores over time after oral administration of THC (solid line; open circles) or PBO (dashed line; closed circles). Means are based on DEQ data from 14 subjects and VAS data from 15 subjects. Error bars represent SEM. ${ }^{*} p<0.05$, two-tailed.

extracted parameter estimates of threat-related amygdala activation were entered into a paired $t$ test (THC vs PBO); significance was set at $p<0.05$, two-tailed. We also calculated Cohen's $d$, an index of effect size, based on the following: Cohen's $d=$ mean $\beta_{\mathrm{PBO}}-$ mean $\beta_{\mathrm{THC}} / \sigma_{\text {pooled }}$, where $\sigma_{\text {pooled }}=\sqrt{ }\left(\sigma_{\mathrm{PBO}}{ }^{2}+\sigma_{\mathrm{THC}}{ }^{2}\right) / 2 ;(\sigma=\mathrm{SD})$. To examine the regional and task-related specificity of THC-mediated effects, we also confirmed activation of primary visual (V1) and motor (M1) cortex [M1, mapped to ipsilateral (left) motor cortex controlling right index finger movement] during the visual-motor task (ON vs OFF) during the placebo and that no differences existed between THC and placebo sessions in terms of V1 and M1 activation using both voxelwise and ROI-based analyses; significance was set liberally at $p<0.05$, uncorrected.

In addition, to obviate bias and for subsequent generation of new hypotheses, we report activation results in brain regions other than the amygdala. Activations to pertinent contrasts (threat vs nonthreat; $\mathrm{PBO}$ vs THC) are shown at a significance height/intensity threshold of $p<0.001$, uncorrected, and extent threshold of clusters with a minimum of 20 contiguous voxels (supplemental Table 1, available at www.jneurosci.org as supplemental material).

Behavioral data analysis. We determined the effects of THC on emotion matching accuracy (percentage correct) and response times (in milliseconds) using a drug (THC, PBO) by condition (threat, nonthreat) ANOVA; significant main effects or drug by condition interactions were followed up with paired $t$ tests (THC vs PBO). Subjective mood/drug effects (DEQ, VAS) were analyzed using two-factor ANOVA with factors drug (THC, PBO) and time (T1-T5). Significant main effect of drug and drug by time interactions were followed with paired $t$ tests (THC vs PBO) to examine the source of significance. Significance for all tests was set at $p<0.05$, two-tailed.

\section{Results}

The participants had a mean $( \pm S D)$ age of $20.8( \pm 2.6)$ years and used marijuana at a mean $( \pm S D)$ frequency of $2.0( \pm 2.4)$ times per month. One-half of the participants were male $(n=8)$, and 13 identified themselves as Caucasian (one African-American, one Asian American, one of mixed ethnicity). Their current use (mean $\pm \mathrm{SD}$ ) of alcohol was $5.4 \pm 3.1$ drinks per week, of caffeine was $6.8 \pm 6.4$ drinks per week, and of tobacco was $12.0 \pm 21.3$ cigarettes per week (six were daily cigarette smokers).

Behavioral results ( $n=14$; data from two subjects are unavailable because of mechanical failure of the response box) show that there was a significant main effect of condition for both accuracy and response time (accuracy: $F_{(1,13)}=5.35, p=0.038$; response time: $\left.F_{(1,13)}=83.00, p=5.24 \times 10^{-7}\right)$, such that subjects were more accurate and faster for the nonthreat compared with the threat condition [accuracy (percentage correct $\pm \mathrm{SD}$ ): nonthreat, $97.3 \pm 6.9 \%$, vs threat, $95.2 \pm 7.2 \% ; t=2.32 ; p=0.04$; response time (in milliseconds $\pm \mathrm{SD}$ ): nonthreat, $1298 \pm 273 \mathrm{~ms}$, vs threat, $\left.1551 \pm 304 \mathrm{~ms} ; t=9.13 ; p=5.14 \times 10^{-7}\right]$. However, there were no significant main effect of drug or drug by condition interactions on accuracy or response times (values of $p>0.05$ ).

Subjective data were missing from two subjects on the DEQ $(n=14)$ and from one subject on the VAS $(n=15)$. THC significantly increased ratings of "feel" drug and feeling "high" [signif- icant drug by time interactions: "feel" $\left(F_{(4,56)}=7.3, p<0.001\right)$; DEQ "high" $\left(F_{(4,56)}=5.0, p=0.002\right)$; and VAS "high" $\left.\left(F_{(4,60)}=6.9, p<0.001\right)\right]$. Follow-up $t$ tests revealed that the mean scores on these measures were significantly higher for THC, relative to placebo, at the following time points: at 90, 210, and $270 \mathrm{~min}$ (all values of $p<0.05$ ), and peaked between the 90 and 210 min (Fig. 1). Thus, the subjective peak effects coincided with fMRI measurement of amygdala reactivity. THC did not significantly change ratings of DEQ "like drug" or "want more" or VAS ratings of "stimulated," "anxious," "sedated," and "hungry."

The fMRI data showed that THC significantly attenuated amygdala activation to threatening faces but had no effect on primary visual and motor activation. In the voxelwise fMRI data analysis, a significant drug by threat interaction (PBO $>$ THC by threat $>$ nonthreat) was localized to the right lateral amygdala [peak MNI coordinates $(36,0,-24) ; F_{(1,30)}=13.92 ; p<0.02$, corrected]. During the placebo session, amygdala activation was greater for threat (fearful, angry) than nonthreat (happy) faces in the right lateral amygdala $[(34,0,-20) ; Z=3.16 ; p=0.02$, corrected] (Fig. $2 A)$, confirming previous findings of amygdala reactivity to threat signals (Phan et al., 2002; Zald, 2003). Notably, however, this threat-related activation was absent during the THC condition, even at lowered significance thresholds (e.g., $p<$ 0.05 , uncorrected) (Fig. 2 B). Compared with placebo, THC significantly attenuated right lateral amygdala activation to threat $[(36,0,-24) ; Z=3.19 ; p<0.02$, corrected] (Fig. 2C). The ROI-based fMRI data analysis was consistent with this, revealing greater right amygdala activation to threat-related (angry/fearful $>$ happy) faces in the placebo than the THC session $[\beta$ weight (in arbitrary units), mean \pm SEM: PBO, $0.21 \pm 0.12$, vs THC, $-0.05 \pm 0.11 ; p<0.05$; Cohen's $d=0.34]$. Moreover, greater amygdala reactivity was observed on the $\mathrm{PBO}$ relative to the THC session in each threat-related condition separately (angry $>$ happy: PBO, $0.15 \pm 0.05$, vs THC, $-0.01 \pm 0.04 ; p<0.05$; fearful $>$ happy: $\mathrm{PBO}, 0.16 \pm 0.07$, vs THC, $-0.08 \pm 0.06 ; p<0.05)$, suggesting that $\mathrm{THC}$ attenuated amygdala reactivity to both angry and fearful faces. To clarify whether THC modulated amygdala reactivity to threat or nonthreat faces, we compared threat and nonthreat conditions separately against the "control" task of matching geometric shapes (which were interspersed between the emotional face blocks). Relative to matching shapes, amygdala reactivity to nonthreat faces was greater during the THC than PBO session (THC, $0.10 \pm 0.08$, vs PBO, $-0.25 \pm 0.14 ; p<$ 0.05 ) (see supplemental Fig. 2, available at www.jneurosci.org as supplemental material). In addition, relative to matching shapes, amygdala reactivity to threat faces was greater during $\mathrm{PBO}$ than THC session (PBO, $0.47 \pm 0.15$, vs THC, $-0.11 \pm 0.12 ; p<0.05$ ) (see supplemental Fig. 2, available at www.jneurosci.org as supplemental material). The pattern of change in amygdala reactivity suggests that the THC effect on amygdala reactivity to threat (vs nonthreat) was primarily driven by attenuation of its response to threat signals (angry, fearful). The THC-mediated increase in amygdala response to signals of nonthreat (i.e., happy faces vs shapes), concurrent with its attenuation to threat-related signals, is an interesting finding that we did not predict a priori, and could be related to the prosocial effects of THC (Foltin and Fischman, 1988; Haller et al., 2004) or its enhanced processing of rewardrelated signals (i.e., happy faces) (Gardner, 2005). Future studies are needed to disentangle the differential effects of THC/canna- 
binoids on appetitive and aversive stimuli. Of note, THC also attenuated threatrelated activation in the left amygdala, but the effect was not significant (PBO, $0.17 \pm$ 0.11 , vs THC, $0.06 \pm 0.15 ; p=0.31$; Cohen's $d=0.19$ ).

During the visual-motor task, V1 and M1 were activated during both the placebo $[\mathrm{V} 1,(-6,-74,0) ; Z=4.64 ; p<$ 0.05 , corrected; left $\mathrm{M} 1,(-48,-12,60)$; $Z=4.64 ; p<0.05$, corrected] and THC sessions $[\mathrm{V} 1,(-8,-78,-2) ; Z=4.64$; $p<0.05$, corrected; left M1, $(-44,-14$, 54); $Z=6.47 ; p<0.05$, corrected]; no significant differences were noted in $\mathrm{V} 1$ and $\mathrm{M} 1$ activation between $\mathrm{PBO}$ and THC sessions. In support, ROI-based fMRI data analysis of $\mathrm{V} 1$ and $\mathrm{M} 1$ activation confirmed a lack of difference between $\mathrm{PBO}$ and THC conditions (V1: PBO, $1.48 \pm$ 0.20 , vs THC, $1.47 \pm 0.18 ; p=0.95 ; \mathrm{M} 1: \mathrm{PBO}, 0.12 \pm 0.12$, vs THC, $0.83 \pm 0.10 ; p=0.63)$.

In post hoc Pearson correlation analyses, we examined the relationship between $(\mathrm{PBO}>\mathrm{THC}$ ) change in right-sided threatrelated amygdala activation (extracted parameter estimates of activation from right amygdala $\mathrm{ROI}$ ) and change ( $\mathrm{THC}>\mathrm{PBO})$ in subjective mood related to drug (DEQ "feel drug" and "drug high"; VAS "high") at the 90, 210, and 270 min time points, at which THC exhibited a significant increase in ratings. The extent of attenuation of right amygdala reactivity by THC was positively related to extent of increase in DEQ "feel drug" by THC at the 210 time point at trend level significance $(r=0.48$; $p<0.06$, twotailed); this correlation should be interpreted cautiously given that the significance is not corrected for the number of correlations analyzed. No other significant associations were observed with DEQ "drug high" or VAS "high" or at the other time points (all values of $p>0.10$ ).

\section{Discussion}

We found that a cannabinoid attenuated limbic reactivity to a social threat stimulus in humans. Using placebo-controlled THC administration and a well validated task to probe threat responses, we found that THC significantly reduced amygdala reactivity to faces conveying threat in recreational marijuana users. The THC-induced attenuation was specific to threatening (vs nonthreatening) stimuli and discretely localized to the amygdala; THC did not affect activity in visual and motor cortex. THC increased subjective reports of drug "high" and "feel drug" but did not affect task performance or induce nonspecific drug effects (e.g., subjective arousal, sedation, anxiety). Reduction of amygdala reactivity to social threat by THC, an exogenous cannabinoid, is consistent with our a priori hypothesis. The current findings fit well with the notion that THC and other cannabinoids may have an anxiolytic role in central mechanisms of fear behaviors.

The THC-mediated effect on amygdala responsivity we describe at the neurobiological level was moderate in size in a relatively small sample population and in the absence of significant changes in subjective anxiety. The moderate dose of THC increased ratings of "drug high" and "feel drug" but, consistent with what has been reported previously, did not increase feelings of anxiety (Chait and Zacny, 1992; Wachtel et al., 2002). Thus, the drug attenuated threat-related amygdala reactivity without con-

\section{Threat vs Non-Threat}

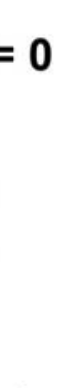

0

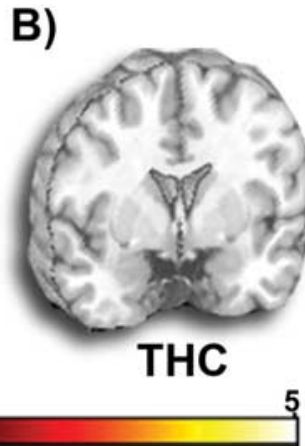

C)

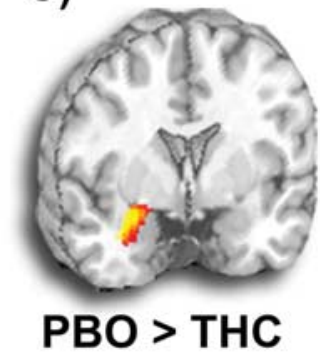

t-score

Figure 2. THC effects on amygdala activation. $\boldsymbol{A}, \boldsymbol{B}$, Statistical $t$ maps overlaid on a canonical brain rendering (MNI coronal ) showing right lateral amygdala activation to threat ( $>$ nonthreat) faces is present during the PBO session but absen during the THC session. C, Statistical $t$ map overlaid on a canonical brain rendering (MNI coronal $y$-plane $=0$ ) showing greater threat-related amygdala reactivity in the PBO relative to the THC session (PBO $>$ THC). For additional information, see Results Statistical $t$ score scale is shown at the bottom of the brain rendering. $R$, Right.

current observable changes in anxiety. The absence of overt conscious anxiolytic activity may be related to several factors. First, amygdala activation as measured by fMRI may be the more relevant indicator of the central effects of THC on conscious threat perception (Adolphs, 2002), and changes in self-reported subjective state may not be relevant to the organism's response to threat. Second, the self-report scales (VAS) used here may not be sensitive to the emotion-relevant and/or fear-specific changes induced by THC, and anxiolytic effects in these healthy, nonanxious subjects may only be apparent with stronger anxiety-inducing stimuli. Third, higher doses or alternative methods of THC administration may be needed to induce effects on anxiety levels (D'Souza et al., 2004). Of note, several other pharmacological fMRI studies using drugs known to have anxiolytic effects have observed significant reduction of amygdala reactivity to conscious and nonconscious threat cues (e.g., angry/fear faces) in the absence of changes in subjective anxiety (Paulus et al., 2005; Harmer et al., 2006) and prosocial effects (Kirsch et al., 2005). Hence, amygdala reactivity may be an important assay of the effects of THC on brain function in relation to threat-related reactivity, despite the absence of self-reported changes in anxiety.

Consistent with findings in nonhumans, the amygdala is critical and particular to threat perception and fear learning in humans (LeDoux, 2000; Zald, 2003; Phelps, 2004; Adolphs et al., 2005). Activation in the amygdala increases with exposure to faces that convey threat, such as fearful and angry faces, but diminished with faces that convey acceptance, such as happy faces (Morris et al., 1996; Whalen et al., 1998). Consistent with this, individuals with social phobias exhibit exaggerated amygdala reactivity to threatening faces (Stein et al., 2002; Phan et al., 2006), whereas those who eagerly engage in social interactions (social fearlessness) show diminished amygdala reactivity to the same types of faces (Meyer-Lindenberg et al., 2005). Amygdala hyperactivity to threatening faces has also consistently been observed in patients with anxiety disorders (Etkin and Wager, 2007). It is important to note that our emotional face matching task and subsequent analysis aimed to compare processing of faces that signal threat (angry, fearful faces) against matched faces that did not signal threat (happy faces). Given that we specifically observed amygdala activation to the threat-related and not to nonthreat, social signals during the placebo session, the effects observed in the THC session can be ascribed distinctly to attenuation of amygdala activation that is specific to threat faces. 
The observation that THC reduced reactivity to threat-related stimuli is consistent with other evidence that cannabinoids play a role in fear and anxiety. Although high doses of THC may appear to increase anxiety (D'Souza et al., 2004; Genn et al., 2004; Viveros et al., 2005), low doses of cannabinoid receptor $\left(\mathrm{CB}_{1}\right)$ agonists, including THC, attenuate anxiety responses in animal models of fear and anxiety, including the elevated plus-maze and social interaction tests (Onaivi et al., 1990; Navarro et al., 1993; Rodriguez de Fonseca et al., 1997; Berrendero and Maldonado, 2002). In contrast, genetic disruption of the $\mathrm{CB}_{1}$ receptor and selective pharmacological $\mathrm{CB}_{1}$ antagonism enhance anxiety responses (Rodriguez de Fonseca et al., 1997; Hajos and Freund, 2002; Haller et al., 2002; Martin et al., 2002). Human recreational cannabis users often report that low doses of the drug produce feelings of calmness and decreased anxiety (Abood and Martin, 1992; Porter and Felder, 2001), and as a result, $\mathrm{CB}_{1}$ receptor and endocannabinoid modulation are currently under study as targets for new treatments of anxiety disorders (Witkin et al., 2005).

At the neuroanatomical and synaptic level, it has been hypothesized that the anxiolytic effects of cannabinoids are mediated via $\mathrm{CB}_{1}$ activation of GABAergic (Katona et al., 2001) or glucocorticoid (Rodriguez de Fonseca et al., 1997) mechanisms within the amygdala. $\mathrm{CB}_{1}$ receptors are densely localized to a distinct population of GABAergic interneurons in the lateral and basal (BLA), but not central (CeA), nuclei of the amygdala (Katona et al., 2001). The baso/lateral amygdala is thought to process aversive sensory stimuli via afferent inputs to central amygdala (LeDoux, 2000). It is also believed that GABAergic neurons in the intercalated nuclei serve as an intermediate relay station to generate feedforward inhibition of central amygdala after activation by basolateral amygdala (Pare and Smith, 1993). The effects of selective $\mathrm{CB}_{1}$ agonists on GABA-mediated inhibitory postsynaptic currents at lateral, but not central, amygdala nuclei may reduce inhibitory tone on basolateral amygdala cells. Thus, $\mathrm{CB}_{1^{-}}$ mediated agonism, by endocannabinoids or exogenous THC, reduces GABA release in BLA interneurons, thereby reducing their inhibition of GABAergic neurons of the intercalated nuclei, which, in turn, increases their inhibition of the pyramidal neurons of the CeA (Katona et al., 2001). In other words, the reduction in inhibitory tone may in turn indirectly reduce anxiety by enhancing the activity of intercalated GABAergic cells that inhibit activation of the central nucleus (Katona et al., 2001). In support, it has been shown that $\mathrm{CB}_{1}$ agonists decrease the excitability of projection neurons in the rat basolateral amygdala (Pistis et al., 2004). In humans, the benzodiazepine, pro-GABAergic anxiolytic agent lorazepam has been shown to attenuate amygdala reactivity to threatening faces (Paulus et al., 2005).

An alternative mechanism by which cannabinoids may exert their anxiolytic effects is via stress-related hormonal systems. Cannabinoids decrease corticotrophin-releasing hormone $(\mathrm{CRH})$ levels in the central nucleus of the amygdala, and decreased CRH levels are associated with decreased aversive stress responses (Rodriguez de Fonseca et al., 1997). In animals, CRHmediated activation of amygdala regulates stress and fear responses to aversive sensory stimuli (Davis, 1992). In humans, endogenous cortisol release has been associated with enhanced amygdala activation to fear-related stimuli (van Stegeren et al., 2007) and low doses of exogenously administered glucocorticoids have been shown to reduce social stress-induced fear in humans (Soravia et al., 2006). Thus, there is evidence for both GABAergic and CRH-mediated mechanisms for the anxiolytic effects of THC, and it is quite possible that $\mathrm{CB}_{1}$ receptor activation may also involve other neurochemical (serotonin, cholecys- tokinin, opioid, etc.) systems relevant to anxiety and fear behaviors (Viveros et al., 2005).

This study should be considered preliminary, and several key related questions remain to be examined. First, although sufficiently powered to detect changes in amygdala reactivity by pharmacologic challenges (Paulus et al., 2005), the sample is insufficient to examine known individual differences in the effects of THC on mood, behavior, and/or brain responses (Wachtel et al., 2002; D'Souza et al., 2004). Second, we chose to administer a low dose of THC in this initial study, and thus, future studies are needed to determine dose-dependent effects of THC on amygdala reactivity to social threat and to examine whether a biphasic (e.g., U-shaped pattern) effect exists at low and high doses, which could help explain differential effects on anxiety levels at varying doses of THC. Moreover, a dose-response study would lend greater support to the inference for a direct effect of THC on amygdala reactivity in relation to dose (Paulus et al., 2005). Third, in addition to amygdala reactivity to social threat conveyed by faces, it would be informative to extend to investigations on the effects of THC on other amygdala-mediated anxiety behaviors such as fear learning in humans, previously shown to be modulated by cannabinoid systems in animals (Marsicano et al., 2002). Fourth, future studies should examine the effects of antagonists at $\mathrm{CB}_{1}$ receptors and exogenous agents that modulate endocannabinoid levels (e.g., rimonabant) on limbic reactivity (Witkin et al., 2005). Fifth, we focused our a priori hypothesis on the amygdala, a region known for its role in threat perception and richly innervated by $\mathrm{CB}_{1}$ receptors, and thus the direct and indirect effects of THC on other regions (e.g., hippocampus, prefrontal cortex) await additional investigation. Lastly, the anatomical ROI used in our planned analyses does not encompass the dorsal extended amygdala (e.g., sublenticular extended amygdala/substantial inominata). Of note, the spatial location and extent of amygdala subregions varies between individuals and standard atlas systems, including the one used here, do not provide information about this interindividual anatomical variability. In addition, the spatial resolution of fMRI and the signal-to-noise ratio in this area of the brain does not enable differentiation of the various subregions or nuclei of the amygdala. These issues limit inferences about the effects of THC on specific amygdala subregions and nuclei (ventral/basolateral nuclei vs dorsal/central nuclei). Resolving this important topic awaits advances in fMRI and other in vivo human functional neuroimaging techniques.

In summary, our data demonstrate a significant and selective impact of THC on amygdala reactivity to social signals of threat in humans. The findings extend the accumulating evidence on cannabinoid modulation of anxiety in humans and nonhumans, and provide evidence for a neuroanatomical site of action for the anxiolytic effects of THC. The current data could prompt the development of new therapies that act on cannabinoid systems to modulate fear behaviors in neuropsychiatric disorders such as social phobia, autism, and schizophrenia, in which social fear or withdrawal, and aberrant reactivity to threat are cardinal features.

\section{References}

Abood ME, Martin BR (1992) Neurobiology of marijuana abuse. Trends Pharmacol Sci 13:201-206.

Adolphs R (2002) Neural systems for recognizing emotion. Curr Opin Neurobiol 12:169-177.

Adolphs R, Gosselin F, Buchanan TW, Tranel D, Schyns P, Damasio AR (2005) A mechanism for impaired fear recognition after amygdala damage. Nature 433:68-72.

Berrendero F, Maldonado R (2002) Involvement of the opioid system in the 
anxiolytic-like effects induced by Delta(9)-tetrahydrocannabinol. Psychopharmacology (Berl) 163:111-117.

Chait LD, Zacny JP (1992) Reinforcing and subjective effects of oral delta 9-THC and smoked marijuana in humans. Psychopharmacology (Berl) 107:255-262.

Chhatwal JP, Davis M, Maguschak KA, Ressler KJ (2005) Enhancing cannabinoid neurotransmission augments the extinction of conditioned fear. Neuropsychopharmacology 30:516-524.

Davis M (1992) The role of the amygdala in fear and anxiety. Annu Rev Neurosci 15:353-375.

D'Souza DC, Perry E, MacDougall L, Ammerman Y, Cooper T, Wu YT, Braley G, Gueorguieva R, Krystal JH (2004) The psychotomimetic effects of intravenous delta-9-tetrahydrocannabinol in healthy individuals: implications for psychosis. Neuropsychopharmacology 29:1558-1572.

Etkin A, Wager TD (2007) Functional neuroimaging of anxiety: a metaanalysis of emotional processing in PTSD, social anxiety disorder, and specific phobia. Am J Psychiatry 164:1476-1488.

Fitzgerald DA, Angstadt M, Jelsone LM, Nathan PJ, Phan KL (2006) Beyond threat: amygdala reactivity across multiple expressions of facial affect. NeuroImage 30:1441-1448.

Folstein MF, Luria R (1973) Reliability, validity, and clinical application of the Visual Analogue Mood Scale. Psychol Med 3:479-486.

Foltin RW, Fischman MW (1988) Effects of smoked marijuana on human social behavior in small groups. Pharmacol Biochem Behav 30:539-541.

Friston KJ, Holmes AP, Worsley KJ, Poline JB, Frith CD, Frackowiak RS (1995) Statistical parametric maps in functional imaging: a general linear approach. Hum Brain Mapp 2:189-210.

Gardner EL (2005) Endocannabinoid signaling system and brain reward: emphasis on dopamine. Pharmacol Biochem Behav 81:263-284.

Genn RF, Tucci S, Marco EM, Viveros MP, File SE (2004) Unconditioned and conditioned anxiogenic effects of the cannabinoid receptor agonist CP 55,940 in the social interaction test. Pharmacol Biochem Behav 77:567-573.

Gur RC, Sara R, Hagendoorn M, Marom O, Hughett P, Macy L, Turner T, Bajcsy R, Posner A, Gur RE (2002) A method for obtaining 3-dimensional facial expressions and its standardization for use in neurocognitive studies. J Neurosci Methods 115:137-143.

Hajos N, Freund TF (2002) Pharmacological separation of cannabinoid sensitive receptors on hippocampal excitatory and inhibitory fibers. Neuropharmacology 43:503-510.

Haller J, Bakos N, Szirmay M, Ledent C, Freund TF (2002) The effects of genetic and pharmacological blockade of the $\mathrm{CB} 1$ cannabinoid receptor on anxiety. Eur J Neurosci 16:1395-1398.

Haller J, Varga B, Ledent C, Barna I, Freund TF (2004) Context-dependent effects of CB1 cannabinoid gene disruption on anxiety-like and social behaviour in mice. Eur J Neurosci 19:1906-1912.

Hariri AR, Tessitore A, Mattay VS, Fera F, Weinberger DR (2002a) The amygdala response to emotional stimuli: a comparison of faces and scenes. NeuroImage 17:317-323.

Hariri AR, Mattay VS, Tessitore A, Fera F, Smith WG, Weinberger DR (2002b) Dextroamphetamine modulates the response of the human amygdala. Neuropsychopharmacology 27:1036-1040.

Hariri AR, Drabant EM, Munoz KE, Kolachana BS, Mattay VS, Egan MF, Weinberger DR (2005) A susceptibility gene for affective disorders and the response of the human amygdala. Arch Gen Psychiatry 62:146-152.

Harmer CJ, Mackay CE, Reid CB, Cowen PJ, Goodwin GM (2006) Antidepressant drug treatment modifies the neural processing of nonconscious threat cues. Biol Psychiatry 59:816-820.

Holmes AP, Friston KJ (1998) Generalisability, random effects and population inference. NeuroImage 7:S754.

Johanson CE, Uhlenhuth EH (1980) Drug preference and mood in humans: diazepam. Psychopharmacology (Berl) 71:269-273.

Kalin NH, Shelton SE, Davidson RJ, Kelley AE (2001) The primate amygdala mediates acute fear but not the behavioral and physiological components of anxious temperament. J Neurosci 21:2067-2074.

Kamprath K, Marsicano G, Tang J, Monory K, Bisogno T, Di Marzo V, Lutz B, Wotjak CT (2006) Cannabinoid $\mathrm{CB}_{1}$ receptor mediates fear extinction via habituation-like processes. J Neurosci 26:6677-6686.

Katona I, Rancz EA, Acsady L, Ledent C, Mackie K, Hajos N, Freund TF (2001) Distribution of $\mathrm{CB}_{1}$ cannabinoid receptors in the amygdala and their role in the control of GABAergic transmission. J Neurosci 21:9506-9518.
Kirk JM, de Wit H (1999) Responses to oral delta9-tetrahydrocannabinol in frequent and infrequent marijuana users. Pharmacol Biochem Behav 63:137-142.

Kirsch P, Esslinger C, Chen Q, Mier D, Lis S, Siddhanti S, Gruppe H, Mattay VS, Gallhofer B, Meyer-Lindenberg A (2005) Oxytocin modulates neural circuitry for social cognition and fear in humans. J Neurosci 25:11489-11493.

Laviolette SR, Grace AA (2006) Cannabinoids potentiate emotional learning plasticity in neurons of the medial prefrontal cortex through basolateral amygdala inputs. J Neurosci 26:6458-6468.

LeDoux JE (2000) Emotion circuits in the brain. Annu Rev Neurosci 23:155-184.

Marsicano G, Wotjak CT, Azad SC, Bisogno T, Rammes G, Cascio MG, Hermann H, Tang J, Hofmann C, Zieglgansberger W, Di Marzo V, Lutz B (2002) The endogenous cannabinoid system controls extinction of aversive memories. Nature 418:530-534.

Martin M, Ledent C, Parmentier M, Maldonado R, Valverde O (2002) Involvement of CB1 cannabinoid receptors in emotional behaviour. Psychopharmacology (Berl) 159:379-387.

Meyer-Lindenberg A, Hariri AR, Munoz KE, Mervis CB, Mattay VS, Morris CA, Berman KF (2005) Neural correlates of genetically abnormal social cognition in Williams syndrome. Nat Neurosci 8:991-993.

Morris JS, Frith CD, Perrett DI, Rowland D, Young AW, Calder AJ, Dolan RJ (1996) A differential neural response in the human amygdala to fearful and happy facial expressions. Nature 383:812-815.

Navarro M, Fernandez-Ruiz JJ, de Miguel R, Hernandez ML, Cebeira M, Ramos JA (1993) An acute dose of delta 9-tetrahydrocannabinol affects behavioral and neurochemical indices of mesolimbic dopaminergic activity. Behav Brain Res 57:37-46.

Onaivi ES, Green MR, Martin BR (1990) Pharmacological characterization of cannabinoids in the elevated plus maze. J Pharmacol Exp Ther 253:1002-1009.

Pare D, Smith Y (1993) The intercalated cell masses project to the central and medial nuclei of the amygdala in cats. Neuroscience 57:1077-1090.

Patel S, Roelke CT, Rademacher DJ, Hillard CJ (2005) Inhibition of restraint stress-induced neural and behavioural activation by endogenous cannabinoid signalling. Eur J Neurosci 21:1057-1069.

Paulus MP, Feinstein JS, Castillo G, Simmons AN, Stein MB (2005) Dosedependent decrease of activation in bilateral amygdala and insula by lorazepam during emotion processing. Arch Gen Psychiatry 62:282-288.

Phan KL, Wager T, Taylor SF, Liberzon I (2002) Functional neuroanatomy of emotion: a meta-analysis of emotion activation studies in PET and fMRI. NeuroImage 16:331-348.

Phan KL, Fitzgerald DA, Nathan PJ, Tancer ME (2006) Association between amygdala hyperactivity to harsh faces and severity of social anxiety in generalized social phobia. Biol Psychiatry 59:424-429.

Phelps EA (2004) Human emotion and memory: interactions of the amygdala and hippocampal complex. Curr Opin Neurobiol 14:198-202.

Pistis M, Perra S, Pillolla G, Melis M, Gessa GL, Muntoni AL (2004) Cannabinoids modulate neuronal firing in the rat basolateral amygdala: evidence for CB1- and non-CB1-mediated actions. Neuropharmacology 46:115-125.

Porter AC, Felder CC (2001) The endocannabinoid nervous system: unique opportunities for therapeutic intervention. Pharmacol Ther 90:45-60.

Rauch SL, Whalen PJ, Shin LM, McInerney SC, Macklin ML, Lasko NB, Orr SP, Pitman RK (2000) Exaggerated amygdala response to masked facial stimuli in posttraumatic stress disorder: a functional MRI study. Biol Psychiatry 47:769-776.

Ressler KJ, Mayberg HS (2007) Targeting abnormal neural circuits in mood and anxiety disorders: from the laboratory to the clinic. Nat Neurosci 10:1116-1124.

Rodriguez de Fonseca F, Carrera MR, Navarro M, Koob GF, Weiss F (1997) Activation of corticotropin-releasing factor in the limbic system during cannabinoid withdrawal. Science 276:2050-2054.

Sethi BB, Trivedi JK, Kumar P, Gulati A, Agarwal AK, Sethi N (1986) Antianxiety effect of cannabis: involvement of central benzodiazepine receptors. Biol Psychiatry 21:3-10.

Soravia LM, Heinrichs M, Aerni A, Maroni C, Schelling G, Ehlert U, Roozendaal B, de Quervain DJ (2006) Glucocorticoids reduce phobic fear in humans. Proc Natl Acad Sci USA 103:5585-5590.

Stein MB, Goldin PR, Sareen J, Zorrilla LT, Brown GG (2002) Increased 
amygdala activation to angry and contemptuous faces in generalized social phobia. Arch Gen Psychiatry 59:1027-1034.

Stenger VA, Boada FE, Noll DC (2000) Three-dimensional tailored RF pulses for the reduction of susceptibility artifacts in $\mathrm{T}\left({ }^{*}\right)(2)$-weighted functional MRI. Magn Reson Med 44:525-531.

Tessitore A, Hariri AR, Fera F, Smith WG, Chase TN, Hyde TM, Weinberger DR, Mattay VS (2002) Dopamine modulates the response of the human amygdala: a study in Parkinson's disease. J Neurosci 22:9099-9103.

Tzourio-Mazoyer N, Landeau B, Papathanassiou D, Crivello F, Etard O, Delcroix N, Mazoyer B, Joliot M (2002) Automated anatomical labeling of activations in SPM using a macroscopic anatomical parcellation of the MNI MRI single-subject brain. NeuroImage 15:273-289.

van Stegeren AH, Wolf OT, Everaerd W, Scheltens P, Barkhof F, Rombouts SA (2007) Endogenous cortisol level interacts with noradrenergic activation in the human amygdala. Neurobiol Learn Mem 87:57-66.

Viveros MP, Marco EM, File SE (2005) Endocannabinoid system and stress and anxiety responses. Pharmacol Biochem Behav 81:331-342.
Wachtel SR, ElSohly MA, Ross SA, Ambre J, de Wit H (2002) Comparison of the subjective effects of Delta(9)-tetrahydrocannabinol and marijuana in humans. Psychopharmacology (Berl) 161:331-339.

Walter B, Blecker C, Kirsch P, Sammer G, Schlienle A, Stark R, Vaitl D (2003) MARINA: an easy to use tool for the creation of MAsks for Region of INterest Analyses. Paper presented at Ninth International Conference on Functional Mapping of the Human Brain. New York, NY, June.

Whalen PJ, Rauch SL, Etcoff NL, McInerney SC, Lee MB, Jenike MA (1998) Masked presentations of emotional facial expressions modulate amygdala activity without explicit knowledge. J Neurosci 18:411-418.

Witkin JM, Tzavara ET, Nomikos GG (2005) A role for cannabinoid CB1 receptors in mood and anxiety disorders. Behav Pharmacol 16:315-331.

Worsley KJ, Marrett P, Neelin AC, Friston KJ, Evans AC (1996) A unified statistical approach for determining significant signals in images of cerebral activation. Hum Brain Mapp 4:58-73.

Zald DH (2003) The human amygdala and the emotional evaluation of sensory stimuli. Brain Res Brain Res Rev 41:88-123. 